\title{
Halting Coronavirus Replication
}

\author{
Kira Smith, MD* \\ Department Clinical and Experimental Medicine, Novara (NO), Italy
}

\section{*Corresponding author}

Kira Smith, MD

Researcher, Independent/MilMed Corporation, Department Clinical and Experimental Medicine, Novara (NO), Italy; Phone:.+I 702-75I-0760;

E-mail: kira-smith@mil-med.com

\author{
Article information \\ Received: July 3I $\mathrm{It}^{\text {st }}$ 2020; Revised: September $2^{\text {nd }}$, 2020; Accepted: September 25 th 2020 ; Published: October $5^{\text {th }}, 2020$
}

Cite this article

Smith K. Halting coronavirus replication. Epidemiol Open J. 2020; 5(I): |3-15. doi: |0. I7|40/EPOJ-5-I| 9

\begin{abstract}
|
Sirtuin 1 (SIRT1) inhibitors can reduce replication of many viruses with certain characteristics similar to those of coronaviruses, while the $p 53$ protein is another important factor in the down-regulation of viral growth. Tenovin is a class of small molecules that inhibit Sirtuin 1 and 2 , in addition to activating the $p 53$ protein, by means of regulating the interactions used by coronaviruses as a self-defense mechanism. By blocking virus growth and continuous replication, with already tested antiviral medicines, the promise of coronavirus disease 2019 (COVID-19) eradication exists.
\end{abstract}

Keywords

COVID-19; Coronavirus replication; SIRT1 inhibitors; $p 53$; Tenovin.

\section{INTRODUCTION |}

\section{How Coronavirus Enters the Host}

$\mathrm{T}$ O date, it is known that the Novel Coronavirus (severe acute respiratory syndrome coronavirus 2 (SARS-CoV-2)) penetrates host cells via the angiotensin converting enzyme 2 (ACE2) receptor, by binding to glycoprotein $\mathrm{S}$, with some differences from what happens with SARS-CoV-1. ${ }^{1}$

From here, you can draw up a list of currently available interventions or known mechanisms of action to reduce the expression of ACE2 activity, in order to avoid (in whole or in part) the entry of the virus into the subject under attack, but not only that. It is also possible that, by lowering its endo-ribonucleic activity to reduce the ability of the virus to reproduce. So, let us see what can be done.

\section{Angiotensin Converting Enzyme 2 Inhibitors: Direct and Indirect Pathways}

We assume that ACE inhibitors (Lisinopril, Quinapril, Captopril) do not reduce ACE2 activity, despite being structurally related and traceable in the same organs, ACE2 has opposing biological effects (vasodilatation, bronchodilatation, activity to compensate for physical stresses, as some particular conditions, like hypoxia); some direct ACE2 antagonists are:

- The small molecule MLN-4760-B and its isomeric MILN4760, much more selective and effective;

- DX600 peptide (and DX-512) that have a nanomolar affinity for ACE2, much more than for ACE (almost null), and competitive and non-competitive inhibition.

The effectiveness and selectivity between these two elements are concentration-related, but at the concentration of 10 $\mu \mathrm{M}, \mathrm{MLN}-4760$ was found to have a better activity profile. ${ }^{2}$

\section{Sirtuin I Inhibitors as Angiotensin Converting Enzyme 2 and Virus Replication Suppressors}

There is also the possibility of acting indirectly, through a Sirtuine group protein, 7 enzymes NAD+ dependent, known as Sirtuin 1(SIRT1).

Expression of ACE2 activity varies with binding to the SIRT1 protein, so by inhibiting SIRT1, we lower ACE2 activity. ${ }^{3}$

Sirtuine are located in the cellular nucleus (SIRT1, SIRT6 and SIRT7), in the cytoplasm (SIRT2), or in the mitochondria (SIRT3, SIRT4 and SIRT5) and are involved in many cellular functions, such as metabolism, the cell cycle, apoptosis, deoxyri- 
bonucleic acid (DNA) repair, etc. Recent studies have found that sirtuine may also have enzymatic functions. They are sensors of changes in the intra- and extracellular environment, generally involved in maintaining human health, but are also implicated in viral replication. Examples of virus replication reduced by Sirtuine inhibitors

- The Tat protein of the human immunodeficiency virus infection (HIV) virus is regulated by SIRT1, whose activity of deacetylase, and vice versa is inhibited by the protein Tat. The transcription of HIV is regulated by SIRT1, by means of Tat deacetylase. SIRT1 preserves the defenses of the virus, throughout its evolution, allowing the recycling of the protein Transcriptional transactivators (Tat), which binds to transactivation response RNA structures (TAR), and the continuous prolongation of messenger ribonucleic acid (mRNA) transcription;

- SIRT1 inhibition (and sometimes even SIRT2) by minor changes to short interfering RNA (siRNA), is known to cause the decrease in replication of additional viruses: flu strains, vesicular stomatitis virus (VSV), Kaposi's Sarcoma-associated Herpes virus (KSHV), Hepatitis B, Hepatitis Cytomegalovirus (CMV), adenovirus, polyomavirus and in some cases the diseases counteract: some types of cancer and Huntington's Chorea, sometimes in combination with antivirals;

- Among the various other viruses that respond by slowing their growth, there is just middle east respiratory syndrome-coronavirus (MERS-Cov). It has been observed that SIR2 (SIRT1 is the human correspondent) acts as the proviral of MERS-CoV in yeast, due to interactions between ORF4a and eukaryotic cells. The YDL042C/SIR2 yeast gene is a suppressor of ORF4a function. When SIRT1 is inhibited by either chemical or genetic manipulation, there is a reduction of MERS-CoV replication. Moreover, ORF4a inhibited SIRT1-mediated modulation of $\mathrm{NF}-x \mathrm{~B}$ signaling, demonstrating a functional link between ORF4a and SIRT1 in mammalian cells. A functional link has been identified between the MERS-CoVORF4a proteins and the YDL042C/SIR2 yeast gene. ${ }^{5}$

- Vesicular stomatitis virus (VSV-SARS-St19) infection is mediated by SARS-CoV-S protein in an ACE2-dependent manner. VSV-SARS-St19 will be useful for analyzing the function of SARS-CoV-S protein and for developing rapid methods of detecting neutralizing antibodies specific for SARS-CoV infection. SIRT1 inhibitors stop the growth of VSV, since the cells' apoptotic response is reduced in cells affected by VSV.

The effectiveness and selectivity between these two elements are concentration-related, but at the concentration of $10 \mu \mathrm{M}, \mathrm{MLN}-4760$ was found to have a better activity profile. ${ }^{2}$

\section{HIF-I $\alpha$ Stabilization PromotesCovid-19 Replication and "Cytokine Storms"}

SIRT1 stabilizes HIF- $1 \alpha$ via direct binding and deacetylation, while SIRT1 depletion or inhibition led to reduced hypoxic HIF- $1 \alpha$ accumulation, accompanied by an increase in HIF- $1 \alpha$ acetylation.
SIRT1-mediated accumulation of HIF- $1 \alpha$ protein led to increased expression of HIF-1 a target genes, including VEGF, GLUT1 and MMP2. HIF-1 $\alpha$ stabilization requires SIRT1 activation.?

Blood monocytes from severe COVID-19 patients present high expression of HIF- $1 \alpha$ in comparison to healthy controls.

The target genes are involved in the glucose transport and glycolytic pathway, were increased in CoV-2-infected monocytes. Stabilization of HIF-1 $\alpha$ increased the expression of ACE2, IL- $1 \beta$, TNF- $\alpha$, IL- 6 , and IFN $\alpha, \beta$, and $\lambda$ in CoV-2-infected monocytes.

Reduced respiration in LPS-activated macrophages is known to increase mitochondrial ROS (mtROS) production, which are strong inducers of HIF-1 $\alpha^{8}$

\section{Coronaviruses Self-Defense Mechanism}

Interacion with SARS-unique domain (SUD) and papain-like protease $\left(\mathrm{PL}^{\mathrm{PRO}}\right)$ against $p 53$.

The strategy developed from SARSCoV and other coronaviruses against host immune recognition system is expressed by a SUD that interacts with its partner cellular E3 ubiquitin ligase ring-finger and $\mathrm{CHY}$ zinc-finger domain-containing 1 (RCHY1) and with papain-like protease $\left(\mathrm{PL}^{\mathrm{PRO}}\right)$. The consequence is a downregulation of $p 53$, involved in the reduction of virus replication. The SARS-CoV papain-like protease is encoded next to SUD within nonstructural protein 3. A SUD-PL ${ }^{\mathrm{PRO}}$ fusion interacts with RCHY1 more intensively and causes stronger $p 53$ degradation than SARS-CoVPL ${ }^{\mathrm{PRO}}$ alone.

\section{How p53 is Important in Coronaviruses Replication}

The $p 53$ protein reduces coronavirus replication, because normalgene activation is involved in the cell's non-specific antiviral defense system. In cells in which $p 53$ is too low, the rate of coronavirus replication is several orders of magnitude higher than that observed in cells in which the $p 53$ is present. ${ }^{7,8}$

\section{Autophagy and Lysosomotropic Agents}

Hypothetic useful drugs in Covid-19 fight also interfere with lysosomal activity and autophagy, interact with membrane stability and alter signalling pathways and transcriptional activity, which can result in inhibition of cytokine production and modulation of certain co-stimulatory molecules. This because Entry of CoVs into the host cells is mainly mediated by the endocytic pathway, meanwhile the autophagy has also been implicated in the viral replication in the cells, a process partly related to the formation of DMV in the host cell.

\section{CONCLUSION}

\section{Hypothetic Perfect Molecule in the Halt of Replication}

There are some small molecules inhibitors of sirtuin 1 and activa- 
tors of p53 protein, which protect it from mdm2-mediated degradation with little effect on p53 synthesis. Histone deacetylase inhibitorscan also indirectly affect the function of some E3 ubiquitin ligases.

In addition, these compounds are autophagy inhibitors and lysosomotropic agents.

\section{Tenovin-I as Hypothetic Perfect Molecule in the Halt of Replication}

Tenovin-1 [Formal name: N-[[[4-(acetylamino)phenyl]amino] thioxomethyl-4-(1,1-dimethylethyl)]-benzamide] is a small molecule inhibitor of sirtuin 1 and sirtuin 2,8 an activator of $p 53$, less toxic of Tenovin-6.

Tenovin- 1 at concentration of $10 \mu \mathrm{M}$ protects $p 53$ from mdm2-mediated degradation with little effect on $p 53$ synthesis.

Histone deacetylase inhibitors like tenovin-1 can also indirectly affect the function of some E3 ubiquitin ligases.

Tenovin- 6 is an analog of tenovin-1 At $10 \mu \mathrm{M}$, this compound is slightly more effective than tenovin- 1 at elevating $p 53$ activity, but it's more toxic.

\section{REFERENCES}

1. Chen Y, Guoa Y, Pana Y, Zhao ZJ. Structure analysis of the receptor binding of 2019-nCoV. Biochemical and Biophysical Research Communications. 2020; 525(1): 135-140. doi: 10.1016/j.bbrc.2020.02.07

2. Li W, Zhang C, Sui J, Kuhn JH, Moore MJ, Luo S, et al. Re- ceptor and viral determinants of SARS-coronavirus adaptation to human ACE2. EMBOJ. 2005; 24: 1634-1643. doi: 10.1038/ sj.emboj. 7600640

3. Clarke NE, Belyaev ND, Lambert DW, Turner AJ. Epigenetic regulation of angiotensin-converting enzyme 2 (ACE2) by SIRT1 under conditions of cell energy stress. Clin Sci (Lond). 2014; 126(7): 507-516. doi: 10.1042/CS20130291

4. Koyuncu E, Budayeva HG, MitevaYV, Ricci DP, Silhavy TJ, Shenk T, et al. Sirtuins are evolutionarily conserved viral restriction factors. mBio. 2014; 5(6): e02249-14. doi: 10.1128/mBio.02249-14

5. Weston S, Matthews KL, Lent R, Vlk A, Haupt R, Kingsbury $\mathrm{T}$, et al. A yeast suppressor screen used to identify mammalian SIRT1 as a proviral factor for Middle East respiratory syndrome coronavirus replication. J Virol. 2019; 93: e00197-19. doi: 10.1128/ JVI.00197-19

6. Fukushi S, Watanabe R, Taguchi F. Pseudotyped vesicular stomatitis virus for analysis of virus entry mediated by SARS coronavirus spike proteins. In: Cavanagh D, eds. $S A R S$ - and Other Coronaviruses. Methods in Molecular Biology (Methods and Protocols); Totowa, NJ, USA: Humana Press; 2008.

7. Smith K. Novel Coronavirus. Hypothesis of Treatment with SIRT1 inhibitors. doi: 10.13140/RG.2.2.35123.99365

8. Ma-Lauer Y, Carbajo-Lozoya J, Hein MY, Müller MA, Deng W, Lei J, et al. p53 down-regulates SARS coronavirus replication and is targeted by the SARS-unique domain and PLpro via E3 ubiquitin ligase RCHY1. PNAS. 2016; 113 (35): 201603435. doi: 10.1073/ pnas. 1603435113 